\title{
Spectrum of impulse control behaviours in Parkinson's disease: pathophysiology and management
}

\author{
Mark John Kelly @ (1, ${ }^{1,2}$ Fahd Baig, ${ }^{3}$ Michele Tao-Ming Hu, ${ }^{2}$ David Okai ${ }^{4,5}$
}

\begin{abstract}
- Additional material is published online only. To view please visit the journal online (http://dx.doi.org/10.1136/ jnnp-2019-322453).
\end{abstract}

${ }^{1}$ School of Medicine, Trinity Centre for Health Sciences, Tallaght University Hospital, Trinity College, Dublin, Ireland ${ }^{2}$ Oxford Parkinson's Disease Centre, Division of Neurology, Nuffield Department of Clinical Neurosciences, Oxford, UK ${ }^{3}$ Molecular and Clinical Sciences Research Centre, London, UK, University of London Saint George's, London, UK

${ }^{4}$ Neuropsychiatry Department, South London and Maudsley NHS Foundation Trust, London, UK

${ }^{5}$ Department of Psychological Medicine, Institute of Psychiatry, Psychology \& Neuroscience, King's College London, London, UK

\section{Correspondence to} Dr Mark John Kelly, School of Medicine, Trinity Centre for Health Sciences, Tallaght University Hospital, Trinity College, Dublin D24 NR04, Ireland; kellym42@tcd.ie

Received 27 January 2020 Revised 13 April 2020 Accepted 14 April 2020 Published Online First 30 April 2020

Check for updates

(C) Author(s) (or their employer(s)) 2020. No commercial re-use. See rights and permissions. Published by BMJ.

To cite: Kelly MJ, Baig F, Hu

MT-M, et al. I Neurol

Neurosurg Psychiatry

2020:91:703-711.

\section{ABSTRACT}

Impulse control behaviours (ICBs) are a range of behaviours linked by their reward-based, repetitive natures. They can be precipitated in Parkinson's disease (PD) by dopamine replacement therapy, often with detrimental consequences for patients and caregivers. While now a well-recognised non-motor feature of treated $P D$, much remains unknown about the influence of risk factors, pathophysiological mechanisms, vulnerability factors for specific types of behaviour and the optimal management strategies. Imaging studies have identified structural and functional changes in striatal and prefrontal brain regions, among others. Gene association studies indicate a role for genetic predisposition to PD-ICB. Clinical observational studies have identified potential modifiable and non-modifiable risk factors. Psychological studies shed light on the neurocognitive domains implicated in PD-ICBs and identify psychosocial determinants that may perpetuate the cycle of impulsive and harm-avoidance behaviours. Based on these results, a range of pharmacological and non-pharmacological management strategies have been trialled in PD-ICBs with varying success. The purpose of this review is to update clinicians on the evidence around the pathophysiology of PD-ICB. We aim to translate our findings into an interpretable biopsychosocial model that can be applied to the clinical assessment and management of individual cases of PD-ICB.

\section{INTRODUCTION}

Impulse control behaviours (ICBs) are a spectrum of behaviours characterised by repetitive, rewardbased actions and subjective loss of control. In their severe form, they are known as impulse control disorders (ICDs). In Parkinson's disease (PD), ICBs can occur as adverse effects of dopamine replacement therapies (DRTs), particularly dopamine agonists (DAs), ${ }^{1}$ with potentially devastating and long-lasting psychosocial effects on patients and caregivers. ${ }^{2}$ There is a strong clinical need to understand the pathophysiology underlying PD-ICB and how to evaluate and manage PD-ICBs in clinical practice.

PD-ICBs are common, although the reported prevalence varies widely $(14 \%-40 \%) .{ }^{1}{ }^{3-6}$ This is likely due to differences in diagnostic criteria, assessment tools ${ }^{7}$ and possibly cultural differences between populations. ${ }^{36}$

The most common PD-ICBs described are as follows:

- Pathological gambling $(\mathrm{PG}) .^{\mathrm{S} 1}$

- Hypersexuality. ${ }^{8}$
- Compulsive shopping (CS). ${ }^{\mathrm{S} 2}$

- Compulsive or binge eating (BE). ${ }^{\mathrm{S} 1}$

- Hobbyism. ${ }^{9}{ }^{10}$

- Punding. $^{9}$

- Dopamine dysregulation syndrome (DDS)/ compulsive medication use. ${ }^{11}$

Other behaviours, sometimes considered subsets of those above, include hoarding ${ }^{12}$ and walkabout. ${ }^{13}$ ICBs more specific to the technological era, such as internet overuse and gaming, ${ }^{S 3}$ are increasingly recognised. This is unsurprising, as a fundamental nature of these technologies is to encourage repetitive use leading to an increased recognition of excessive use as a problem in the wider population. ${ }^{14}$ The specific form of internet overuse varies, and, depending on the driver, may include aspects of hobbyism, punding or even gambling and hypersexuality. The incidence of these behaviours is likely to increase with time as technology is increasingly used by older generations.

A phenomenon that specialists may recognise from clinical practice is that PD-ICBs exists on a broad spectrum of severity, ranging from a change in premorbid traits that are still considered within the limits of normal behaviour to severe disorders of high frequency and intensity (see figure 1).

Diagnostic Statistical Manual (DSM-) and DSMaligned diagnostic criteria exist for individual ICBs (see references listed above). While this specificity is useful in research, these criteria risk giving the erroneous impression that PD-ICBs exist as a binary entity-in which patients either meet or do not meet criteria for 'caseness'. Conversely, screening questionnaires such as the Questionnaire for Impulse Control Disorders in Parkinson's Disease (QUIP) ${ }^{13}$ and the Minnesota Impulsive Disorders Interview $(\mathrm{MIDI})^{15}$ may capture most cases without differentiating the innocuous from the dangerous. This categorical approach fails to recognise the dimensional nature of the spectrum where 'subsyndromal' PD-ICDs may have an observable impact on patients without meeting strict diagnostic criteria. This has additionally been a limitation of many PD-ICB prevalence studies. ${ }^{17}$ Attempts to address this dimensional approach include the QUIP rating scale (QUIP-RS) and Parkinson's Impulse Control Scale (PICS). ${ }^{16}{ }^{17}$ Recognition of PD-ICB as a spectrum will help to risk-stratify patients, target interventions appropriately and assess response to such interventions in future observational and treatment studies.

The objectives of this review are to as follows:

1. Update clinicians on what is known about the pathophysiology of PD-ICB, presenting a 


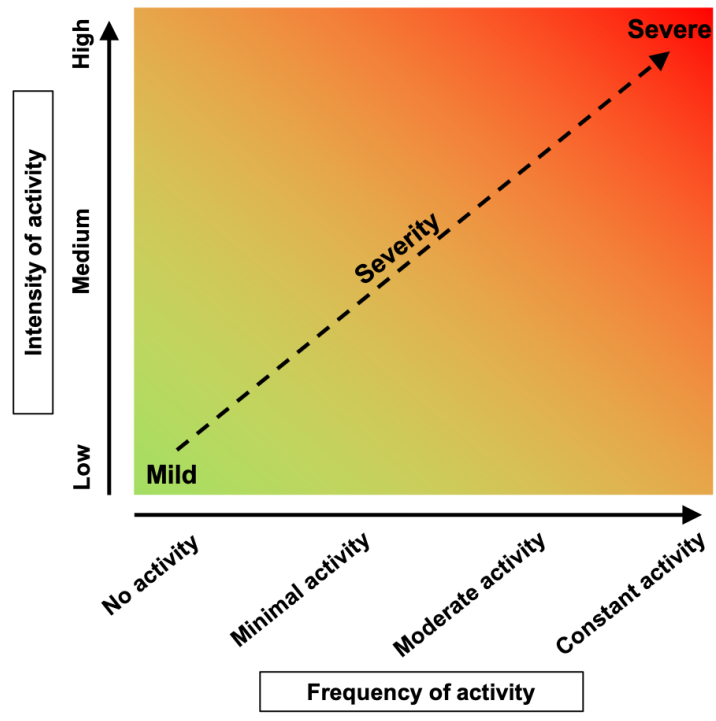

Figure 1 Conceptual illustration of the Parkinson's disease with impulse control behaviour (PD-ICB) spectrum. PD-ICBs exist on a spectrum of severity determined by both the frequency of the impulsive activity and the intensity or magnitude of that activity, ie, the impact that it has on patients and caregivers. At the mild end of the spectrum, these behaviours may be considered 'within normal limits' and have little or no impact. At the severe end of the spectrum, behaviours typically meet the diagnostic criteria for PD-ICD. In the middle of the spectrum, however, behaviours may not meet these criteria and be considered 'subsyndromal', but still have a significant impact that may require monitoring or intervention. Adapted from Parkinson's Impulse Control Scale rating scale and results from Baig and colleagues (2019). ${ }^{29}$

comprehensive and interpretable biopsychosocial model that takes into account neurochemical, neural-network, psychological and epidemiological evidence to date.

2. Highlight how to assess and manage PD-ICBs using pharmacological and non-pharmacological approaches, underpinned through application of this model.

Terminology regarding PD-ICBs differs between studies and specialist centres. For example, the term 'impulsive and compulsive behaviours' ${ }^{18}$ is also used. While harm avoidance is included in the psychological formulation of PD-ICB, there is little evidence to suggest the behaviours denote 'compulsions', which are defined as behaviours specifically targeted to relieve anxiety. ${ }^{\mathrm{S} 1}$ Furthermore, the strongest evidence to date favours ICBs representing a 'devaluation of future rewards' or immediate gratification (also known as myopia for the future). ${ }^{19}$ Therefore, in this review, the term 'impulse control behaviour' and the following terminologies are used:

- Impulse control disorder (ICD/PD-ICD): An ICB that fits DSM or published DSM-aligned criteria, that is, 'Syndromal' ICD. The cornerstone of such criteria is that the ICB sufficiently impacts on social and/or occupational functioning.

- Subsyndromal impulse control disorder: An ICB that falls short of the criteria for ICD. In the case of PD, this specifically refers to a change in behaviour; either an exacerbation of previous behaviours or their novel occurrence, thought related to the introduction of DRT.

- ICB/PD-ICB: The full spectrum of ICB, as defined above, including not only syndromal ICDs but also subsyndromal ICDs. N.B. this includes the full range of disinhibitory psychopathologies listed above including hobbyism and punding.

\section{Pathophysiology}

Animal models, imaging, neuropsychometric and observational clinical studies provide evidence on mechanisms underpinning PD-ICB. Evidence from the study of ICBs in the healthy population, particularly PG, have been extrapolated to and investigated in PD. While describing all the complex theories proposed to explain impulsivity would be beyond the scope of this review, we aim to examine some of the more comprehensive neurochemical, neural-network, psychological and clinical models of PD-ICB.

\section{Neurochemical modelling}

The role of dopamine

While impulsivity is a complex and heterogeneous concept (see the Psychological modelling section), reward and punishment learning, mediated primarily by dopamine, are recognised as key components of impulsivity in $\mathrm{PD}-\mathrm{ICB},{ }^{\mathrm{S}}$ providing a useful model to examine the role of dopamine in PD-ICB.

Dopamine receptor subtypes have varying effects and patterns of distribution throughout the basal ganglia. D3 receptors are expressed preferentially in the ventral striatum (vs), linked to reward processing. ${ }^{\mathrm{S}}$ D1 and D2 receptors have been proposed, in a computational model, to mediate motor response inhibition, demonstrated by procedural learning tasks. ${ }^{20}$ Phasic dopaminergic bursts in the D1-mediated direct (excitatory, 'Go') pathway are released in response to positive stimuli. Dips or pauses in dopamine release in the D2-mediated indirect (inhibitory, 'NoGo') pathway occur in response to negative outcomes. This model has been consistently replicated clinically: medicated patients with PD demonstrate impaired negative-reinforcement learning and an enhanced response to positive reinforcement, with the opposite observed in individuals with unmedicated PD. ${ }^{21} 22 \mathrm{~S} 6$

Modern DAs demonstrate relative selectivity for D2-type (D2, D3, D4) receptors over D1 types (D1, D5). ${ }^{\text {S7 }}$ DAs, therefore, may overstimulate D3-mediated reward circuits and excessively disinhibit the D2-mediated indirect pathway. These effects may be less pronounced with levodopa therapy, which demonstrates a less selective, and more physiological receptor activation pattern.

In animal models of PD-ICB, pramipexole enhances delay discounting, a marker of impulsive decision-making. ${ }^{\text {S8 }}$ D2 and D3 agonists stimulate reward-seeking behaviour in rodents at intermediate doses. ${ }^{S 9}$ However, in these experimental models, these effects also occur in control animals, not just PD models. Furthermore, in the latter study, ${ }^{\mathrm{S} 9} \mathrm{D} 1$-specific agonists and higher doses of D2 and D3 agonists actually induced reward aversion, inconsistent with the clinical models described above. It is thus likely that preclinical models cannot yet recreate all the complex neurochemical processes underlying PD-ICB. ${ }^{\mathrm{S} 10}$

Nuclear imaging studies demonstrate lower levels of D2/D3 receptor binding ${ }^{23-25}$ and reduced dopamine transporter (DAT) availability $^{26}$ in the vs of PD-ICB subjects. The latter has been shown in longitudinal analyses to predate the introduction of DRT. $^{27}$ S11 These findings may be explained by greater levels of vs dopamine activity (either due to enhanced release and/ or reduced uptake). Thus, D2/D3 receptors are 'overdosed' by the addition of DRT, increasing the potential for rewarddriven behaviours. ${ }^{\text {S12 }}$ Conversely, PD-ICB patients may have a greater burden of dopaminergic circuit denervation, potentially 
explaining why patients with more advanced disease are at greater risk of PD-ICB. ${ }^{28} 29$

The role of genetics in dopamine signalling

Kraemmer and colleagues ${ }^{30}$ found the heritability of PD-ICB to be $57 \%$, suggesting a genetic predisposition in some individuals. Although, only a screening questionnaire (QUIP) was used for diagnosis. Heterogeneity in neurotransmitter metabolism may explain why some individuals are at greater risk of PD-ICB. The findings of seven candidate gene association studies comparing PD patients with and without PD-ICB are shown in table 1 . Polymorphisms of DRD1, DRD2, DRD3, SLC22A1 and DDC, all involved in dopamine signalling and metabolism, have been associated with increased risk of PD-ICB. A polymorphism of DRD4 was associated with changes in gambling task performance on levodopa. ${ }^{31}$ Genetic panels may be useful in predicting PD-ICB risk. ${ }^{30}$ However, results are variable and contradictory, likely due to differences in diagnostic criteria, ethnicity and candidate gene selection. Large-scale, multicentre, genome wide-analysis studies are warranted to investigate the role of genetic risk in PD-ICBs.

Other neurotransmitters

Serotonin, norepinephrine, glutamate and opioids have been implicated in addictive behaviours such as PG and substance use disorder (SUD). For a detailed review, see previous work. ${ }^{32}$ Genes involved in neurotransmitter signalling (GRIN2B; glutamate, HTR2A; serotonin and OPRK1; opioids) have been implicated in PD-ICB risk (see table 1). Other than these gene association studies, however, there has been little study into the role of other neurotransmitters in PD-ICB. Modest and sometimes conflicting results for the use of selective serotonin reuptake inhibitors (SSRIs), ${ }^{\mathrm{S} 13}$ selective norepinephrine reuptake inhibitors (SNRIs), ${ }^{\text {S14 }}$ opioid receptor antagonists ${ }^{\mathrm{S} 15}$ and N-methyl-Daspartate (NMDA-) glutamate receptor antagonists ${ }^{33}$ S16-S18 are discussed in more detail under the Management section.

\section{Neural-network modelling}

Impulsivity and reward-based decision-making are mediated by a complex neural-network interconnected by mesocortical and mesolimbic circuits. Clinical imaging studies have attempted to demonstrate whether anatomical and/or functional changes in neural-networks predispose certain individuals to ICB within the general population and in PD.

Reward circuits project from the ventral tegmental area (VTA) to cortical and subcortical areas. ${ }^{\text {S19 }}$ Disruption of these circuits has been implicated in ICDs such as PG and SUD. ${ }^{32}$ The specific areas implicated vary according to imaging modalities used.

Such studies have also been used to investigate a neuralnetwork correlate for increased risk of PD-ICB. Voxel-based morphometry has identified patterns such as cortical thickening of the anterior cingulate cortex (ACC) ${ }^{S 20}$ and cortical thinning of the orbitofrontal cortex (OFC), ${ }^{\mathrm{S} 21}$ associated with PD-ICBs. However, such findings are inconsistently replicated and this modality may not be sensitive to subtle or functional changes. ${ }^{34}$

Brain activity studies such as functional MRI (fMRI) may be more sensitive to functional changes in reward circuitry. These studies are small in size, few in number and vary in their methods and results. A recent meta-analysis ${ }^{35}$ of such studies found that the most consistently implicated brain activity changes between PD-ICB and PD-non-ICB individuals were the following:

- Hyperactivity of the vs. vs dopamine release is triggered by unexpected reward stimuli, an important initial step in reward learning. An intact vs, with greater dopaminergic
Table 1 Genetic polymorphisms investigated in PD-ICB

\begin{tabular}{|c|c|c|}
\hline Gene & Associated & Not associated \\
\hline \multicolumn{3}{|l|}{ DRD3 } \\
\hline$r s 6280$ & $\begin{array}{l}\text { Lee et al, } 2009^{570} \\
\text { Krishnamoorthy et al, } \\
2016^{571}\end{array}$ & $\begin{array}{l}\text { Zainal Abidin et al, } 2015^{572} \\
\text { McDonell et al, } 2018^{573} \\
\text { Redenšek et al, 2019 }\end{array}$ \\
\hline rs3732783 & & Zainal Abidin et al, $2015^{572}$ \\
\hline \multicolumn{3}{|l|}{ GRIN2B } \\
\hline rs1806201 & & $\begin{array}{l}\text { Lee et al, } 2009^{570} \\
\text { Krishnamoorthy et al, } \\
2016^{571} \\
\text { Redenšek et al, } 2019^{574}\end{array}$ \\
\hline rs7301328 & $\begin{array}{l}\text { Lee et al, } 2009^{570} \\
\text { Zainal Abidin et al, 2015 } 272\end{array}$ & \\
\hline rs1019385 & & Lee et al, $2009^{570}$ \\
\hline \multicolumn{3}{|l|}{$S L C 22 A 1$} \\
\hline rs628031 & Redenšek et al, $2019^{574}$ & \\
\hline \multicolumn{3}{|l|}{ COMT } \\
\hline$r s 4680$ & & $\begin{array}{l}\text { Vallelunga et al, 2012 } 2^{575} \\
\text { Redenšek et al, } 2019^{574}\end{array}$ \\
\hline rs165815 & & Redenšek et al, $2019^{574}$ \\
\hline \multicolumn{3}{|l|}{ DRD1 } \\
\hline rs4867798 & Zainal Abidin et al, $2015^{572}$ & \\
\hline$r s 4532$ & Zainal Abidin et al, $2015^{572}$ & \\
\hline rs265981 & & Zainal Abidin et al, $2015^{572}$ \\
\hline \multicolumn{3}{|l|}{ DRD2 } \\
\hline rs1800497 (Taq1A) & $\begin{array}{l}\text { Zainal Abidin et al, } 2015^{572} \\
\text { McDonell et al, } 2018^{573}\end{array}$ & $\begin{array}{l}\text { Lee et al, 2009 } 570 \\
\text { Vallelunga et al, } 2012^{575} \\
\text { Kraemmer et al, } 2016^{30}\end{array}$ \\
\hline rs1799732 & & Redenšek et al, $2019^{574}$ \\
\hline rs1801028 & & Redenšek et al, $2019^{574}$ \\
\hline rs104894220 & & Zainal Abidin et al, $2015^{572}$ \\
\hline rs144999500 & & Zainal Abidin et al, $2015^{572}$ \\
\hline$r s 6277$ & & McDonell et al, $2018^{573}$ \\
\hline \multicolumn{3}{|l|}{$D D C$} \\
\hline rs3837091 & Kraemmer et al, $2016^{30}$ & Redenšek et al, $2019^{574}$ \\
\hline rs921451 & & Redenšek et al, $2019^{574}$ \\
\hline rs1451375 & Kraemmer et al, $2016^{30}$ & \\
\hline \multicolumn{3}{|l|}{ MAOB } \\
\hline rs1799836 & & Redenšek et al, $2019^{574}$ \\
\hline \multicolumn{3}{|l|}{ SLC6A3 } \\
\hline 3'UTR 40 bp VNTR & & Vallelunga et al, $2012^{575}$ \\
\hline rs393795 & & Redenšek et al, $2019^{574}$ \\
\hline rs6347 & & Redenšek et al, $2019^{574}$ \\
\hline rs104209 & & Redenšek et al, $2019^{574}$ \\
\hline \multicolumn{3}{|l|}{ SLC6A4 } \\
\hline 5-HTTLPR & & Lee et al, $2009^{570}$ \\
\hline \multicolumn{3}{|l|}{ SLC22A1 } \\
\hline rs628031 & & Redenšek et al, $2019^{574}$ \\
\hline \multicolumn{3}{|l|}{ SLC7A5 } \\
\hline rs 1060253 & & Redenšek et al, $2019^{574}$ \\
\hline rs1060257 & & Redenšek et al, $2019^{574}$ \\
\hline \multicolumn{3}{|l|}{$S L C 18 A 2$} \\
\hline Rs14240 & & Redenšek et al, $2019^{574}$ \\
\hline \multicolumn{3}{|l|}{$S V 2 C$} \\
\hline rs1423099 & & Redenšek et al, $2019^{574}$ \\
\hline \multicolumn{3}{|l|}{$H T 2 R A$} \\
\hline rs6313 & & $\begin{array}{l}\text { Krishnamoorthy et al, } \\
2016^{571}\end{array}$ \\
\hline
\end{tabular}

Continued 


\begin{tabular}{|c|c|c|}
\hline Gene & Associated & Not associated \\
\hline rs1800443 & & Zainal Abidin et al, $2015^{572}$ \\
\hline \multicolumn{3}{|l|}{ DRD5 } \\
\hline rs144132215 & & Zainal Abidin et al, $2015^{572}$ \\
\hline \multicolumn{3}{|l|}{ HTR2A } \\
\hline rs6313 & $\begin{array}{l}\text { Lee et al, 2012*576 } \\
\text { Kraemmer et al, 2016 }\end{array}$ & \\
\hline \multicolumn{3}{|l|}{ OPRK1 } \\
\hline rs702764 & Kraemmer et al, $2016^{30}$ & \\
\hline \multicolumn{3}{|l|}{ ADRA2C } \\
\hline rs 76337672 & & Kraemmer et al, $2016^{30}$ \\
\hline
\end{tabular}

This table summarises the findings of eight candidate gene association studies investigating polymorphisms potentially associated with PD-ICB, according to whether or not the study found an association.

*Borderline association found across whole group ( $p=0.055)$, significant association among lower levodopa equivalent dose (LED) patients $(\mathrm{p}=0.011)$.

activity, may be more sensitive to the 'overdose' of DRT, ${ }^{S 12}$ as mentioned in the Neurochemical modelling section.

- Hyperactivity of the OFC. Dopaminergic activity of the OFC is thought to mediate reward processing and decisionmaking in goal-directed learning. ${ }^{\text {S22 }}$ The OFC may be particularly vulnerable to the tonic stimulation of dopamine receptors by DAs. ${ }^{523}$

- Hypoactivity of the ACC. Reduced ACC activity may impair the perseveration and premeditation required to mitigate impulsive decision-making. ${ }^{\text {S24 }}$

Most imaging studies are cross-sectional and unable to infer causality in the correlations identified. Longitudinal studies are required to identify whether changes in reward circuitry represent a predisposition towards impulsivity or a response to chronic exposure to DA or PD-ICB itself.

Comparison of baseline volumetric MRI in drug-naïve patients did not differentiate between those who later developed PD-ICB $(n=42)$ and PD controls in a case-control study nested within the longitudinal Parkinson Progressive Markers Initiative (PPMI) cohort. ${ }^{\mathrm{S} 25}$ However, another longitudinal study ${ }^{36}$ using restingstate fMRI found several differences in baseline connectivity that were later associated with greater risk of PD-ICB (increased salience network (OFC) connectivity, reduced central executive network (left supramarginal gyrus) and central executive network (left precuneus, right middle temporal gyrus) connectivity). Coupling between salience networks and central executive networks also correlated with greater PD-ICB severity. This study was small $(\mathrm{n}=30,15$ with PD-ICB) and relatively short in duration (36-month follow-up). Only resting-state fMRI was assessed, whereas fMRI during reward-based tasks may be more sensitive to reward-circuit changes. Nevertheless, this and the DAT studies discussed previously ${ }^{27}$ S11 suggest that at least some neural-network changes precede the introduction of DRT and onset of PD-ICB.

DTI studies postulate a role for white-matter denervation in impulsivity. ${ }^{\text {S26 }}$ S27

Larger, prospective functional imaging studies are needed to better investigate the role of heterogeneity in neural-networks and the clinical utility of imaging in predicting PD-ICB risk.

\section{Psychological modelling}

Increased understanding of the neuropsychology of ICBs such as PG and SUD may shed light on the formulation of ICB, the role of dopamine and the predilection towards ICB among certain patients with PD. Here, we outline psychological models of (1) reward seeking and impulsivity and (2) harm avoidance.

\section{Impulsivity}

Impulsivity can be divided into several, not mutually exclusive domains, according to the psychometric tests through which they measured. ${ }^{37}$ In general, these are conceptualised as:

1. Motor impulsivity (response inhibition): Acting without thinking. Most commonly measured by $\mathrm{Go} / \mathrm{NoGo}^{\mathrm{S} 28}$ and Start-Stop Reaction tasks. ${ }^{\text {S29 }}$

2. Decision-making impulsivity: Decision-making involves several cognitive domains including executive function, reward processing and punishment sensitivity. Impulsive individuals appear to make riskier decisions, disproportionately incentivised by positive reward and desensitised to negative consequences. Measured, in part, by gambling tasks. ${ }^{\mathrm{S} 0 \mathrm{~S} 31}$

3. Choice impulsivity: Closely related to decision-making; impulsive individuals have difficulty in delaying gratification despite greater rewards in Delay-Discounting tasks. ${ }^{532}$

4. Reflection impulsivity: Insufficient information gathering when making decisions. Measured by information sampling tasks. ${ }^{533}$

PD-ICBs can be modelled using these domains, although questions still remain regarding their direct translation into pathological behaviours (see review by Dawson and colleagues ${ }^{18}$ ). While the sensitivity and specificity of such tests appear valid, their role as discriminators of severity remains unclear. While some inconsistencies may be explained by variations in methodology, there currently appear limitations to the real-world applicability (or 'ecological validity') ${ }^{534}$ of such paradigms. Such challenges are replicated in other conditions where executive dysfunction may pose a clinical problem and require psychometric evaluation, for example, traumatic brain injury. ${ }^{\mathrm{S} 35}$

PD-ICB patients score higher than PD controls for motor impulsivity, non-planning (choice) impulsivity and attention (reflection impulsivity) on the Barratt Impulsiveness Scale ${ }^{\mathrm{S} 36}$ and on the Delay-Discounting Task, specific to choice impulsivity. ${ }^{28}$ As described in the Neurochemical modelling section, untreated patients with PD demonstrate impaired reward learning and intact reversal learning, whereas dopaminergic treatment reverses this pattern. ${ }^{21}$ Drug-naïve PD patients also demonstrate reduced novelty-seeking behaviours compared with healthy controls. ${ }^{38}$ While this might suggest that patients with PD, in the absence of DRT, are less impulsive than the general population, there is little evidence to indicate a difference in PD-ICB prevalence of between drug-naïve PD patients and healthy controls. ${ }^{39}$ DAs have been shown to interfere with reward prediction error in patients with PD. Excessive dopaminergic stimulation will interpret rewards as 'better than expected', encouraging behaviour through incentive sensitisation. ${ }^{40}$

It is possible that psychological profiles differ between PD-ICBs. For instance, hypersexuality was associated with poorer performance than PG and BE on Stroop tests believed to represent inhibitory control. ${ }^{\mathrm{S} 37} \mathrm{PG}$ and CS have been associated with greater novelty-seeking and CS with greater choice impulsivity than other PD-ICBs. ${ }^{28}$

\section{Harm avoidance}

Impulsivity is generally seen as a novelty-seeking behaviour. ${ }^{\mathrm{S} 38}$ However, it is increasingly recognised that actions in PD-ICB are also driven by harm avoidance. While often considered dimensional opposites, it is likely the relationship is more nuanced than 
this, with both concepts sharing a loss of inhibition and 'lack of control', with an inability to learn from adverse outcomes. ${ }^{37}$

PD-ICB patients perform more poorly on a temporal binding task, indicating loss of 'sense of agency', also known as sense of control, ${ }^{\mathrm{S} 39}$ and demonstrate more harm avoidance than healthy controls. ${ }^{21}{ }^{540}$ It has been theorised that the goal of a PD-ICB is not only to seek a reward or 'high' but relieve dysphoria or a 'low' (eg, feelings of guilt, shame, loneliness, low mood). Various models have been proposed including the idea of (hedonic) homeostatic dysregulation, ${ }^{11}$ applicable to DDS, where evidence suggests the majority take additional medication in anticipatory fear of an 'off period dysphoria'. ${ }^{19}$

Other psychological domains have been implicated in PD-ICB with mixed evidence. For example, some studies have found deficits in executive functions compared with PD controls, ${ }^{41537}$ while others have not. ${ }^{42} 43$

\section{Clinical and demographic modelling}

Numerous studies have investigated clinical and demographic risk factors for PD-ICB. ${ }^{1628294445}$ Clinical modelling may identify patients in whom DA therapy should be avoided or modified.

DA use is the most significant risk factor for PD-ICB. ${ }^{129}$ One of the largest prospective studies of PD-ICB to date found DA use increased PD-ICB risk by about 50\% over levodopa alone. This may be an underestimate considering the high baseline rate of PD-ICB in their population. ${ }^{46}$

For a list of risk factors associated with PD-ICB, see table 2. These factors, divided into potentially modifiable and nonmodifiable, are supported by variable levels of evidence. The term potentially modifiable is used as it cannot be stated with certainty that modification of these risk factors will result in a lower PD-ICB risk. For example, the association between smoking and alcohol consumption and $\mathrm{PD}-\mathrm{ICB}^{146}{ }^{47}$ may represent non-modifiable tendencies towards addictive behaviours, as current smoking has also been associated with PD-ICBs. ${ }^{541}$ Depression and anxiety ${ }^{28} 46$ are considered potentially modifiable as they have readily available therapies. In a cognitive behavioural therapy (CBT) trial addressing PD-ICB, both anxiety and depressive scores improved significantly, ${ }^{48}$ although the direction of any causal relationship between these disorders and PD-ICBs is unclear. Apathy is considered non-modifiable since it potentially represents greater levels of mesolimbic denervation $^{\mathrm{S42}}$ and lacks well-validated therapies.

A meta-analysis of 15 case-control studies, including 999 PD-ICD patients and 3507 PD-non-ICB controls, identified that DA use, DA levodopa daily equivalent dose (LEDD), total LEDD, amantadine use, PD duration, younger age, male sex and smoking were associated with greater PD-ICB risk. Levodopa LEDD, motor symptom severity and cognition were not. ${ }^{49}$ However, this study identified PD-ICBs using screening questionnaires rather than more specific in-depth interviews. ${ }^{17}$ Studies using semistructured interviews have identified associations with levodopa use, ${ }^{1}$ levodopa LEDD $^{28}$ and motor symptom severity. ${ }^{28}{ }^{29}$ The roles of age and sex are disputed by some of these studies ${ }^{28}{ }^{29}$ but supported by others. ${ }^{1} \mathrm{Few}^{50}$ have found an association between PD-ICBs and cognitive impairment but most exclude patients with dementia. Hence, the results of this meta-analysis should not be considered definitive.

An important finding is that PD-ICBs appear to be a DA-LEDD dependant effect, ${ }^{49}$ contrasting with earlier evidence ${ }^{28}$ but in concordance with more recent studies. ${ }^{51}$ The role of amantadine in PD-ICBs is controversial, as discussed later. Male sex and
Table 2 Clinical and demographic factors potentially associated with PD-ICB

\begin{tabular}{|c|c|c|}
\hline & Non-modifiable & Potentially modifiable \\
\hline \multirow{4}{*}{$\begin{array}{l}\text { Medication-related } \\
\text { factors }\end{array}$} & & DA use* \\
\hline & & Levodopa uset \\
\hline & & $\begin{array}{l}\text { Medication dose } \\
\text { Total LEDD* } \\
\text { DA LEDD* }\end{array}$ \\
\hline & & $\begin{array}{l}\text { Other medications } \\
- \text { Amantadine }^{*}\end{array}$ \\
\hline \multirow[t]{3}{*}{ Disease-related factors } & Disease duration* & \\
\hline & $\begin{array}{l}\text { Motor severity } \\
\text { MDS-UPDRS-III† } \\
\text { MDS-UPDRS-II } \\
\text { Hoehn and Yahrt }\end{array}$ & \\
\hline & $\begin{array}{l}\text { Cognitive impairment } \dagger \\
\text { Presence of RBD }\end{array}$ & \\
\hline
\end{tabular}

\begin{tabular}{lll} 
& & \multicolumn{1}{c}{$\begin{array}{c}\text { Depression } \\
\text { Anxiety }\end{array}$} \\
& Apathy & \\
\hline Patient factors & Age* $^{*}$ & \\
\hline & Sex $^{*}$ & Smoking history \\
& & Alcohol use \\
\hline Social factors & Family history of ICB & \\
\hline & Marital status & \\
\hline
\end{tabular}

'Potentially modifiable' implies possible avenues for treatment. Withdrawal and reductions in DRT are known to reduce PD-ICB risk. While depression, anxiety, smoking and alcohol use are treatable risk factors, evidence is lacking on whether treating these features improve outcome in PD-ICB. Symptomatic treatments are available for RBD and apathy but we would speculate that the presence of these syndromes represents a PD phenotype more prone to PD-ICB, rather than a modifiable risk factor.

*Associated with PD-ICB in the meta-analysis by Liu and colleagues. ${ }^{49}$ tNot associated with PD-ICB in the meta-analysis by Liu and colleagues. ${ }^{49}$ DA, dopamine agonist; DRT, dopamine replacement therapy; PD-ICB, Parkinson's disease with impulse control behaviour.

younger age are well-described risk factors for ICB in the general population.

Additional risk factors have been described. Patients with a family history of ICB appear to be at greater risk of PD-ICB after the initiation of DRT, ${ }^{1}$ and smoking and alcohol use have been hypothesised to represent a greater risk among those with premorbid ICB traits. $^{28}$

Apathy, a non-motor feature of PD, was associated with PD-ICB in two large-scale longitudinal analyses. ${ }^{29} 46$ This is interesting as apathy is often considered the 'opposite' of impulsivity and is modulated by dopaminergic therapies. ${ }^{S 43}$ S44 It may be that dysfunction of reward circuits cause a susceptibility to DRT-induced PD-ICB. Alternatively, PD-ICB patients' disinterest in activities outside of their addiction or behaviour may present as a 'pseudo-apathy'.

A correlation between PD-ICBs and motor complications of DRT has been identified in some studies ${ }^{28} 2952$ but not others. ${ }^{50}$ It has been proposed that PD-ICBs and dyskinesia represent end products of similar DRT-induced signalling processes, the former in the vs and the latter in the dorsal striatum. ${ }^{53}$

Whether or not rapid eye movement-sleep behaviour disorder (RBD), considered to represent prodromal parkinsonism, is associated with PD-ICB remains uncertain. A meta-analysis including 10 studies $(n=2781)$ found a positive association between the 
two. ${ }^{54}$ However, the majority of included studies diagnosed RBD on a screening questionnaire. Of the three studies using gold-standard polysomnography, ${ }^{S 45-s 47}$ only one ${ }^{S 45}$ found an association between the PD-ICB and RBD.

Social factors such as being unmarried ${ }^{146}$ and not reaching tertiary education ${ }^{46}$ have been associated with PD-ICB risk, although inconsistently. ${ }^{29}$ One study found tertiary education to be associated with a higher PD-ICB risk. ${ }^{548}$ Social background is important in the formulation of addictive behaviours, particularly gambling, in the general population, ${ }^{\$ 49}{ }^{550}$ but there has been little investigation of social determinants of PD-ICB. It has been theorised, ${ }^{55}$ with some qualitative evidence, ${ }^{29}$ that psychosocial stressors (eg, social isolation) have a predisposing and perpetuating effect in PD-ICB, and that supportive social networks may be protective.

Subtle differences in associated factors have been noted between PD-ICB subtypes (PG with older age, BE with lower rates of anxiety, hypersexuality and CS with greater levodopa doses). ${ }^{28}$ Though, to our knowledge, there has been little attempt to replicate such findings in other observational studies. ${ }^{16294445}$ Determining risk of different PD-ICB subtypes would undoubtedly be useful in clinical practice but require larger sample sizes. The shift to severity scales may allow greater ease of correlation analysis.

Clinicians should bear in mind the risk factors listed in table 2 when considering DA therapy in patients with PD and monitoring for PD-ICB. However, it should be stressed that no risk factors have been associated strongly enough to be considered an absolute contraindication to DA and evidence remains mixed for many factors.

\section{Management}

Because DA use is the greatest predictor of PD-ICB, the goldstandard 'treatment' is to avoid DA in those at greatest risk or to withdraw the medication if PD-ICBs occur. This is reasonable as it is increasingly recognised that DAs are less efficacious, have less tolerable side effects and confer a lower quality of life than levodopa. ${ }^{51}$ The following should be considered:

- Dopamine-agonist withdrawal syndrome or low mood following dose tapering can make it difficult to cease DA treatment. ${ }^{19} \quad 552$ Some patients may require a long-term low dose of DA to prevent apathy or other psychological sequelae.

- Non-DA therapies including levodopa and possibly even deep brain stimulation (DBS) can cause PD-ICB. ${ }^{553}$ Though DBS been reported to improve PD-ICBs in most cases, as discussed in the Management section.

- PD-ICB can persist $\mathrm{S}^{\mathrm{S} 4}$ or 'relapse ${ }^{29}$ in syndromal and subsyndromal forms, even after withdrawal of DA. ${ }^{554}$

Therefore, other management options are needed to complement or even replace DA withdrawal. Appropriate application of these measures requires thorough clinical assessment and effective risk stratification of PD-ICBs.

\section{Clinical assessment}

PD-ICBs are increasingly recognised as a dimensional spectrum of behaviour severity and not a categorical diagnosis ${ }^{19}$ (see figure 1). This has led to the development of clinical assessment tools aimed not only at PD-ICB diagnosis but at grading severity according to its psychosocial impact. Examples include the following:

1. Questionnaire on Impulsivity in Parkinson's-Rating Scale (QUIP-RS). ${ }^{16}$
A 28-point patient-completed or clinician-completed questionnaire. It has been independently validated in individuals with advanced $\mathrm{PD}^{\mathrm{S5}}$ and used as an outcome measure in clinical trials. ${ }^{\text {S15 }}$ Like the QUIP screening tool from which it originates, the QUIP-RS is highly sensitive but non-specific for diagnostic purposes. S56 $^{\text {. }}$

2. Ardouin Scale of Behaviour in Parkinson's Disease (ASBPD). ${ }^{56}$

This semistructured interview evaluates a range of hypodopaminergic and hyperdopaminergic psychological complications in PD, including PD-ICB. It has been validated and used in a clinical trial. ${ }^{57}$ The clinimetrics for the overall scale were acceptable but the internal consistency measures, inter-rater reliability and test-retest reliability for many of the ICB-specific assessments were low. ${ }^{558}$ It is designed for use by psychiatrists and psychologists familiar with PD and takes about an hour to perform, perhaps limiting its use in routine practice.

3. Parkinson's Impulse Control Scale (PICS). ${ }^{17}$

This more recently designed scale uses a semistructured interview to grade behaviours for the full spectrum of PD-ICBs, on the basis of both intensity and impact. The semistructured design complements normal clinical assessment, allowing for input from patients, caregivers and clinicians. It can be performed quickly but requires an experienced clinician. It likely has a higher sensitivity to detecting DDS compared with the QUIP-RS and ASBPD by specifically enquiring about offperiod dysphoria. The validation study demonstrated excellent inter-rater reliability and fair test-retest reliability at 6 months, potentially representing true clinical fluctuations.

These scales have been subject to two systematic reviews. ${ }^{7}$ S59 Aside from those listed above, several more scales exist which apply only to specific ICB subtypes. The QUIP-RS and ASBPD met the threshold to be 'recommended' in a Movement Disorder Society review for clinical use. ${ }^{7}$ The PICS, while 'suggested', now likely also meets the threshold for 'recommended' given its more widespread use. ${ }^{29}$

While it may be assumed that a higher severity score requires more urgent intervention, there are insufficient longitudinal data to correlate present severity score with future risk of deterioration. PD-ICB severity can change significantly over relatively short periods of 1-2 years, with risks of both deterioration and relapse. ${ }^{29}$ Further study is warranted to assess the use of PD-ICB rating scales in risk stratifying patients to direct management.

\section{Pharmacological management}

No pharmacological agents are currently licensed for the treatment of PD-ICB. Several agents have been trialled with very modest results:

- In a randomised controlled trial (RCT), naltrexone did not improve the severity of PD-ICD measured by clinician-rated Clinical Global Impression of Change (CGI) but did reduce the severity as measured by the patient-completed QUIPRS. ${ }^{S 15}$ While warranting further investigation, a major clinical benefit seems unlikely from these results alone.

- Amantadine is used in the treatment of levodopa-induced dyskinesia. ${ }^{560}$ If dyskinesia and PD-ICBs represent similar signalling processes in different striatal regions, it could be theorised that amantadine may mitigate the impulsive effects of DRT. One small placebo-controlled study ${ }^{\mathrm{S16}}$ and another non-controlled study by the same group ${ }^{\mathrm{S} 17}$ found amantadine to be beneficial in the treatment of PG in PD. However, the rate of adverse effects was high and observational studies 
appear to indicate that amantadine use is actually associated with a greater risk of PD-ICB. ${ }^{33}$ S18

- The SSRI citalopram $^{\text {S13 }}$ and the SNRI atomoxetine $^{\text {S14 S61 }}$ demonstrated improvement in psychometric measures of impulsivity in small trials of patients with PD, but have not been trialled in patients with PD-ICB.

- Other: Evidence on other anti-ICB medications comes only from case reports and series. In a systematic review of such cases, sodium valproate demonstrated the most consistently positive results. $^{\text {S62 }}$

To date, no pharmacological agents have sufficient evidence for the treatment of PD-ICB.

Non-pharmacological management

Patients with PD are already subjected to a high pill-burden and significant adverse effects. Therefore, non-pharmacological interventions for non-motor symptoms such as PD-ICBs are being increasingly explored. These include the following:

- Psychotherapy.

As outlined above, psychosocial determinants are important in the formulation of PD-ICB. Healthier coping strategies may break the cycle of novelty-seeking and harm avoidance, mitigating the behaviours' impact. One $\mathrm{RCT}^{48}$ found that 28 patients receiving immediate CBT demonstrated significantly greater improvement in PD-ICB severity measured by CGI and improvements in anxiety and depression levels compared with 17 wait-listed controls, who subsequently demonstrated response to CBT 6 months later. ${ }^{57}$ Consideration of CBT for PD-ICBs is recommended by the National Institute for Health and Care Excellence (NICE). ${ }^{\text {S63 }}$ CBT may provide a useful adjunct or primary therapy in treating PD-ICB and warrants larger trials. ${ }^{58}$

DBS.

Despite conflicting case reports and series ${ }^{\mathrm{S} 53}$ on whether STN-DBS has beneficial or detrimental effects on PD-ICB, a systematic review ${ }^{\mathrm{S} 64}$ and prospective observational studies with extended follow-up periods ${ }^{59} \mathrm{S65}$ report an improvement in PD-ICBs in most patients. This seemingly contradicts experimental evidence that STN activity is inhibitory to impulsivity, and functional inhibition of the STN by DBS should increase risky decision-making. ${ }^{566}$ However, it appears that any such effect is likely superseded by the 'antiimpulsive' benefits of medication reduction. ${ }^{567}$ Furthermore, some studies found that DBS itself reduces the severity of PD-ICB, independent of medication changes. ${ }^{60}$ One longitudinal study found a significant reduction in the prevalence of PG and hypersexuality but only a trend towards this effect in CS and an increase in punding cases. Certain personality traits also increased risk of persisting or de novo PD-ICB. BE has also been reported to persist following STN-DBS. ${ }^{59}$ So STN-DBS may have differing effects according to PD-ICB subtype.

While DBS appears to have beneficial effects for most PD-ICB patients, clinicians should be aware of reported cases of existing PD-ICBs worsening, remaining unchanged or developing de novo. ${ }^{\mathrm{S}}{ }^{4}$ Such cases of DBS-associated PD-ICBs are theorised to be due to spread of current from the dorsolateral motor region of the STN to the closely associated ventromedial limbic region. ${ }^{568}$ Therefore, adjustments in electrode configuration to direct the electrical field away

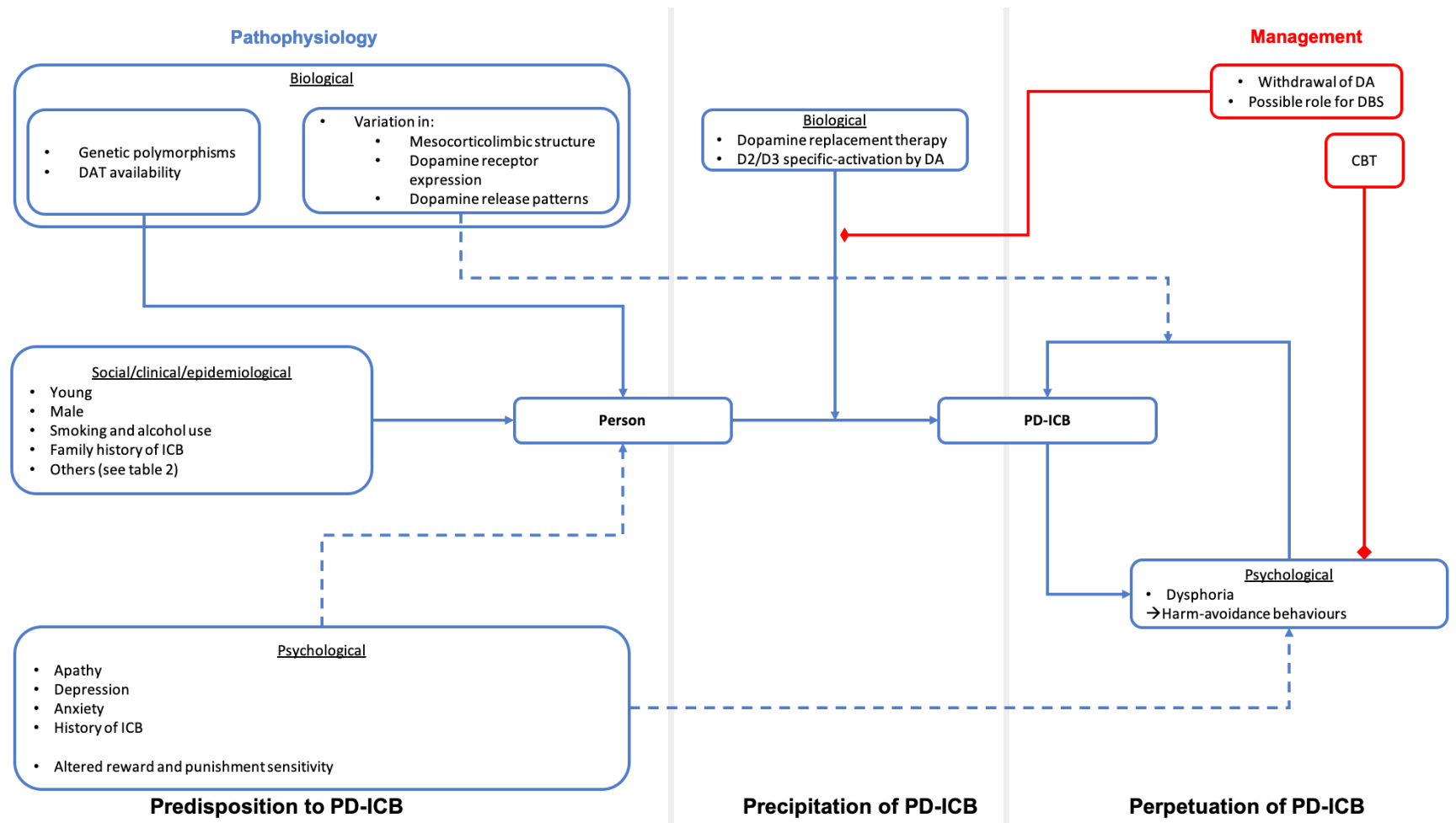

Figure 2 Biopsychosocial model of the pathophysiology and management of PD-ICBs. This simplified model summarises the biological, psychological and social mechanisms that may be implicated in the predisposition, precipitation and perpetuation of PD-ICBs, and how these factors can be targeted in the management of PD-ICBs. Pathophysiological mechanisms are highlighted in blue. Management options are highlighted in red. Dashed arrows signify factors which have been associated with PD-ICBs, although the direction of this relationship is unclear. These factors may therefore have a role in predisposition, perpetuation or neither. DAT, dopamine transporter; DBS, deep brain stimulation; CBT, cognitive behavioural therapy; ICB, impulse control behaviour; PD, Parkinson's disease. 
from these regions may be effective in avoiding or reversing these effects. ${ }^{569}$ Patients may also experience a transient increase in impulsive behaviours in the acute phase after the insertion of electrodes, typically lasting less than a year. ${ }^{59}$ If future guidelines are to suggest DBS as a management option for PD-ICB, attention should be given to these mixed results, the importance of electrode placement and configuration, and close patient follow-up. An RCT of STN-DBS in PD-ICB would help to direct such guidance.

\section{Summary and biopsychosocial model}

PD-ICBs are characterised by subjective loss of control and exist on a broad spectrum of severity. The evidence presented in this review supports the roles of biological, psychological and social factors which may predispose patients to PD-ICBs and perpetuate these behaviours.

Figure 2 illustrates a biopsychosocial model of PD-ICB pathophysiology and management. This model is by no means definitive as much of the pathophysiology remains unclear. However, by combining the most salient findings from neurochemical, imaging, psychological and clinical studies creates a clearer picture of PD-ICB formulation. Biological factors such as genetic polymorphisms, D2/D3-receptor and DAT availability may predispose certain patients to PD-ICB. Other biological changes in dopaminergic circuitry and metabolism have been associated with PD-ICB, and some of these changes may predate the introduction of DRT. Therefore, these factors may have a role in predisposition and/or perpetuation or may simply be biomarkers of PD-ICBs or DRT therapy. The same can goes for psychological comorbidities such as apathy, depression and anxiety.

PD-ICBs can be difficult to manage and evidence on treatments is lacking. This model supports a holistic management strategy, combining pharmacological and non-pharmacological therapies aimed at reducing the severity of PD-ICBs and improving quality of life for patients and caregivers.

\section{Key points}

- PD-ICBs exist across a broad spectrum of severity, ranging from traits that may be considered 'within normal limits' to pathological ICD of extreme intensity, occurring in $14 \%-40 \%$ of patients with PD.

- Dysfunction in reward circuits projecting from the VTA to cortical and subcortical areas, and heterogeneity in dopamine signalling, have been proposed to mediate PD-ICB.

- DA use, DA-LEDD, total LEDD, amantadine use, PD duration, younger age, male sex and smoking, among other factors, have been associated with greater risk of PD-ICB, with genetic risk factors emerging.

- Subtle differences may exist between PD-ICB subtypes. For instance, in psychological profile, clinical risk factors and response to DBS. Such observations warrant replication and further investigation.

- First-line management should include gradual dose reduction and complete withdrawal of DAs where possible. Nonpharmacological approaches include psychotherapy/CBT and possibly DBS.

Contributors MJK searched the literature and wrote the first draft of the manuscript. FB, MT-MH and DO critically revised each version of the manuscript and contributed additional references. MJK is the guarantor of the manuscript.

Funding The authors have not declared a specific grant for this research from any funding agency in the public, commercial or not-for-profit sectors.

Competing interests MJK received a honorarium for a lecture delivered to the Parkinson Voice Project non-profit organisation, Dallas, Texas, USA, on 'Impulsivity and Creativity in Parkinson's' in October 2018. MT-MH receives funding from the Parkinson's UK Monument Discovery Award, Oxford Biomedical Research Centre, University of Oxford, National Institute for Health Research, Michael J Fox Foundation, H2020 European Union, GE Healthcare and the PSP Association. She is on the consultancy advisory board for Biogen and Roche Pharmaceutical companies and is a consultant for CuraSen Therapeutics.

Patient consent for publication Not required.

Provenance and peer review Commissioned; externally peer reviewed.

\section{ORCID iD}

Mark John Kelly http://orcid.org/0000-0001-7214-5039

\section{REFERENCES}

1 Weintraub D, Koester J, Potenza MN, et al. Impulse control disorders in Parkinson disease: a cross-sectional study of 3090 patients. Arch Neurol 2010;67:589-95.

2 Leroi I, Harbishettar V, Andrews M, et al. Carer burden in apathy and impulse control disorders in Parkinson's disease. Int J Geriatr Psychiatry 2012;27:160-6.

3 Fan W, Ding H, Ma J, et al. Impulse control disorders in Parkinson's disease in a Chinese population. Neurosci Lett 2009:465:6-9.

4 Poletti M, Logi C, Lucetti C, et al. A single-center, cross-sectional prevalence study of impulse control disorders in Parkinson disease: association with dopaminergic drugs. $J$ Clin Psychopharmacol 2013;33:691-4.

5 Sáez-Francàs N, Martí Andrés G, Ramírez N, et al. [Clinical and psychopathological factors associated with impulse control disorders in Parkinson's disease]. Neurologia 2016;31:231-8.

6 Joutsa J, Martikainen K, Vahlberg T, et al. Effects of dopamine agonist dose and gender on the prognosis of impulse control disorders in Parkinson's disease. Parkinsonism Relat Disord 2012;18:1079-83.

7 Evans AH, Okai D, Weintraub D, et al. Scales to assess impulsive and compulsive behaviors in Parkinson's disease: critique and recommendations. Mov Disord 2019;34:791-8.

8 Voon V, Hassan K, Zurowski M, et al. Prevalence of repetitive and reward-seeking behaviors in Parkinson disease. Neurology 2006;67:1254-7.

9 O'Sullivan SS, Evans AH, Lees AJ. Punding in Parkinson's disease. Pract Neurol 2007;7:397-9.

10 Averbeck BB, O'Sullivan SS, Djamshidian A. Impulsive and compulsive behaviors in Parkinson's disease. Annu Rev Clin Psychol 2014;10:553-80.

11 Giovannoni G, O'Sullivan JD, Turner K, et al. Hedonistic homeostatic dysregulation in patients with Parkinson's disease on dopamine replacement therapies. J Neurol Neurosurg Psychiatry 2000;68:423-8.

12 O'Sullivan SS, Djamshidian A, Evans AH, et al. Excessive hoarding in Parkinson's disease. Mov Disord 2010;25:1026-33.

13 Weintraub D, Hoops S, Shea JA, et al. Validation of the questionnaire for impulsivecompulsive disorders in Parkinson's disease. Mov Disord 2009;24:1461-7.

14 Kuss DJ, Griffiths MD, Karila L, et al. Internet addiction: a systematic review of epidemiological research for the last decade. Curr Pharm Des 2014;20:4026-52.

15 Antonini A, Chaudhuri KR, Boroojerdi B, et al. Impulse control disorder related behaviours during long-term rotigotine treatment: a post hoc analysis. Eur J Neurol 2016;23:1556-65.

16 Weintraub D, Mamikonyan E, Papay K, et al. Questionnaire for Impulsive-Compulsive disorders in Parkinson's Disease-Rating scale. Mov Disord 2012;27:242-7.

17 Okai D, Askey-Jones S, Mack J, et al. Parkinson's Impulse-Control scale for the severity rating of Impulse-Control behaviors in Parkinson's disease: a semistructured clinical assessment tool. Mov Disord Clin Pract 2016;3:494-9.

18 Dawson A, Dissanayaka NN, Evans A, et al. Neurocognitive correlates of Medicationinduced addictive behaviours in Parkinson's disease: a systematic review. Eur Neuropsychopharmacol 2018;28:561-78.

19 Okai D, Samuel M, Askey-Jones S, et al. Impulse control disorders and dopamine dysregulation in Parkinson's disease: a broader conceptual framework. Eur J Neurol 2011;18:1379-83.

20 Frank MJ, Seeberger LC, O'reilly RC. By carrot or by stick: cognitive reinforcement learning in parkinsonism. Science 2004;306:1940-3.

21 Bódi N, Kéri S, Nagy H, et al. Reward-learning and the novelty-seeking personality: a between- and within-subjects study of the effects of dopamine agonists on young Parkinson's patients. Brain 2009;132:2385-95.

22 Frank MJ, Samanta J, Moustafa AA, et al. Hold your horses: impulsivity, deep brain stimulation, and medication in parkinsonism. Science 2007;318:1309-12.

23 Payer DE, Guttman M, Kish SJ, et al. [ $\left.{ }^{11} \mathrm{C}\right]-(+)-$ PHNO PET imaging of dopamine $D(2 / 3)$ receptors in Parkinson's disease with impulse control disorders. Mov Disord 2015;30:160-6.

24 Stark AJ, Smith CT, Lin Y-C, et al. Nigrostriatal and Mesolimbic $D_{2 / 3}$ Receptor Expression in Parkinson's Disease Patients with Compulsive Reward-Driven Behaviors. J Neurosci 2018;38:3230-9.

25 Lee J-Y, Seo SH, Kim YK, et al. Extrastriatal dopaminergic changes in Parkinson's disease patients with impulse control disorders. J Neurol Neurosurg Psychiatry 2014;85:23-30. 
26 Voon V, Rizos A, Chakravartty R, et al. Impulse control disorders in Parkinson's disease: decreased striatal dopamine transporter levels. J Neurol Neurosurg Psychiatry 2014;85:148-52.

27 Vriend C, Nordbeck AH, Booij J, et al. Reduced dopamine transporter binding predates impulse control disorders in Parkinson's disease. Mov Disord 2014;29:904-11.

28 Voon V, Sohr M, Lang AE, et al. Impulse control disorders in Parkinson disease: a multicenter case--control study. Ann Neurol 2011;69:986-96.

29 Baig F, Kelly MJ, Lawton MA, et al. Impulse control disorders in Parkinson disease and RBD: a longitudinal study of severity. Neurology 2019;93:e675-87.

30 Kraemmer J, Smith K, Weintraub D, et al. Clinical-Genetic model predicts incident impulse control disorders in Parkinson's disease. J Neurol Neurosurg Psychiatry 2016;87:1106-11.

31 Eisenegger C, Knoch D, Ebstein RP, et al. Dopamine receptor D4 polymorphism predicts the effect of L-dopa on gambling behavior. Biol Psychiatry 2010;67:702-6.

32 Potenza MN, Review PMN. Review. The neurobiology of pathological gambling and drug addiction: an overview and new findings. Philos Trans $R$ Soc Lond B Biol Sci 2008;363:3181-9.

33 Weintraub D, Sohr M, Potenza MN, et al. Amantadine use associated with impulse control disorders in Parkinson disease in cross-sectional study. Ann Neurol 2010;68:963-8

34 Ricciardi L, Lambert C, De Micco R, et al. Prospective analysis of morphological markers of development of impulsive compulsive behaviors in de novo Parkinson's disease. Mov Disord 2017;32:1057-8.

35 Santangelo G, Raimo S, Cropano M, et al. Neural bases of impulse control disorders in Parkinson's disease: a systematic review and an ale meta-analysis. Neurosci Biobehav Rev 2019;107:672-85.

36 Tessitore A, De Micco R, Giordano A, et al. Intrinsic brain connectivity predicts impulse control disorders in patients with Parkinson's disease. Mov Disord 2017:32:1710-9.

37 Fineberg NA, Chamberlain SR, Goudriaan AE, et al. New developments in human Neurocognition: clinical, genetic, and brain imaging correlates of impulsivity and compulsivity. CNS Spectr 2014;19:69-89.

38 Baig F, Lawton MA, Rolinski M, et al. Personality and addictive behaviours in early Parkinson's disease and REM sleep behaviour disorder. Parkinsonism Relat Disord 2017;37:72-8.

39 Antonini A, Siri C, Santangelo G, et al. Impulsivity and compulsivity in drug-naïve patients with Parkinson's disease. Mov Disord 2011;26:464-8.

40 Voon V, Pessiglione M, Brezing C, et al. Mechanisms underlying dopamine-mediated reward bias in compulsive behaviors. Neuron 2010;65:135-42.

41 Voon V, Reynolds B, Brezing C, et al. Impulsive choice and response in dopamine agonist-related impulse control behaviors. Psychopharmacology 2010;207:645-59.

42 Djamshidian A, O'Sullivan SS, Sanotsky Y, et al. Decision making, impulsivity, and addictions: do Parkinson's disease patients jump to conclusions? Mov Disord 2012;27:1137-45.

43 Leroi I, Andrews M, McDonald K, et al. Apathy and impulse control disorders in Parkinson's disease: a direct comparison. Parkinsonism Relat Disord 2012;18:198-203.
44 Callesen MB, Weintraub D, Damholdt MF, et al. Impulsive and compulsive behaviors among Danish patients with Parkinson's disease: prevalence, depression, and personality. Parkinsonism Relat Disord 2014;20:22-6.

45 Stanzione P, Antonini A, Bonuccelli U, et al. A 2-year non-interventional study to assess the prevalence of impulsive-compulsive behaviors (ICBS) in an outpatient Italian Parkinson's disease (PD) population (ICARUS). Neurol Sci 2015;36:S201.

46 Antonini A, Barone P, Bonuccelli U, et al. ICARUS study: prevalence and clinical features of impulse control disorders in Parkinson's disease. J Neurol Neurosurg Psychiatry 2017;88:317-24.

47 Joutsa J, Martikainen K, Vahlberg T, et al. Impulse control disorders and depression in Finnish patients with Parkinson's disease. Parkinsonism Relat Disord 2012;18:155-60

48 Okai D, Askey-Jones S, Samuel M, et al. Trial of CBT for impulse control behaviors affecting Parkinson patients and their caregivers. Neurology 2013;80:792-9.

49 Liu B, Luo W, Mo Y, et al. Meta-Analysis of related factors of impulse control disorders in patients with Parkinson's disease. Neurosci Lett 2019;707:134313.

50 Bastiaens J, Dorfman BJ, Christos PJ, et al. Prospective cohort study of impulse control disorders in Parkinson's disease. Mov Disord 2013;28:327-33.

51 Corvol J-C, Artaud F, Cormier-Dequaire F, et al. Longitudinal analysis of impulse control disorders in Parkinson disease. Neurology 2018;91:e189-201.

52 Biundo R, Weis L, Abbruzzese G, et al. Impulse control disorders in advanced Parkinson's disease with dyskinesia: the ALTHEA study. Mov Disord 2017;32:1557-65

53 Voon V, Napier TC, Frank MJ, et al. Impulse control disorders and levodopa-induced dyskinesias in Parkinson's disease: an update. Lancet Neurol 2017;16:238-50.

54 Lu H-T, Shen Q-Y, Zhao Q-Z, et al. Association between REM sleep behavior disorder and impulsive-compulsive behaviors in Parkinson's disease: a systematic review and meta-analysis of observational studies. J Neurol 2020;267:331-40.

55 Delaney M, Leroi I, Simpson J, et al. Impulse control disorders in Parkinson's disease: a psychosocial perspective. J Clin Psychol Med Settings 2012;19:338-46.

56 Ardouin C, Chéreau I, Llorca P-M, et al. [Assessment of hyper- and hypodopaminergic behaviors in Parkinson's disease]. Rev Neurol 2009;165:845-56.

57 Okai D, Askey-Jones S, Samuel M, et al. Predictors of response to a cognitive behavioral intervention for impulse control behaviors in Parkinson's disease. Mov Disord 2015;30:736-9.

58 Koychev I, Okai D. Cognitive-Behavioural therapy for non-motor symptoms of Parkinson's disease: a clinical review. Evid Based Ment Health 2017;20:15-20.

$59 \operatorname{Kim}$ A, Kim YE, Kim H-J, et al. A 7-year observation of the effect of subthalamic deep brain stimulation on impulse control disorder in patients with Parkinson's disease. Parkinsonism Relat Disord 2018;56:3-8.

60 Rossi PJ, De Jesus S, Hess CW, et al. Measures of impulsivity in Parkinson's disease decrease after DBS in the setting of stable dopamine therapy. Parkinsonism Relat Disord 2017;44:13-17. 